\title{
LA-UR-17-26420
}

Approved for public release; distribution is unlimited.

Title: $\quad$ Automated Blazar Light Curves Using Machine Learning

Author(s): $\quad$ Johnson, Spencer James

Intended for: $\quad$ Talk at Internal Meeting Report

Issued: 
Disclaimer:

Los Alamos National Laboratory, an affirmative action/equal opportunity employer, is operated by the Los Alamos National Security, LLC for the National Nuclear Security Administration of the U.S. Department of Energy under contract DE-AC52-06NA25396. By approving this article, the publisher recognizes that the U.S. Government retains nonexclusive, royalty-free license to publish or reproduce the published form of this contribution, or to allow others to do so, for U.S. Government purposes. Los Alamos National Laboratory requests that the publisher identify this article as work performed under the auspices of the U.S. Department of Energy. Los Alamos National Laboratory strongly supports academic freedom and a researcher's right to publish; as an institution, however, the Laboratory does not endorse the viewpoint of a publication or guarantee its technical correctness. 
Automated Blazar Light Curves Using Machine Learning Spencer Johnson

Post-Master's, U. of Cambridge

Tom Vestrand

ISR-2

UNCLASSIFIED 


\section{Personal Background}

- Post-Master's Student in Mathematical Physics from the University of Cambridge

- Preparing for PhD in Physics at UIUC.

- Came to LANL to get experience with data analysis and experimental hardware. 


\section{Background on Problem}

- Blazars are a type of active galactic nucleus which emit relativistic jets in line with the Earth.

- Blazars emit outbursts of energy across the electromagnetic spectrum.

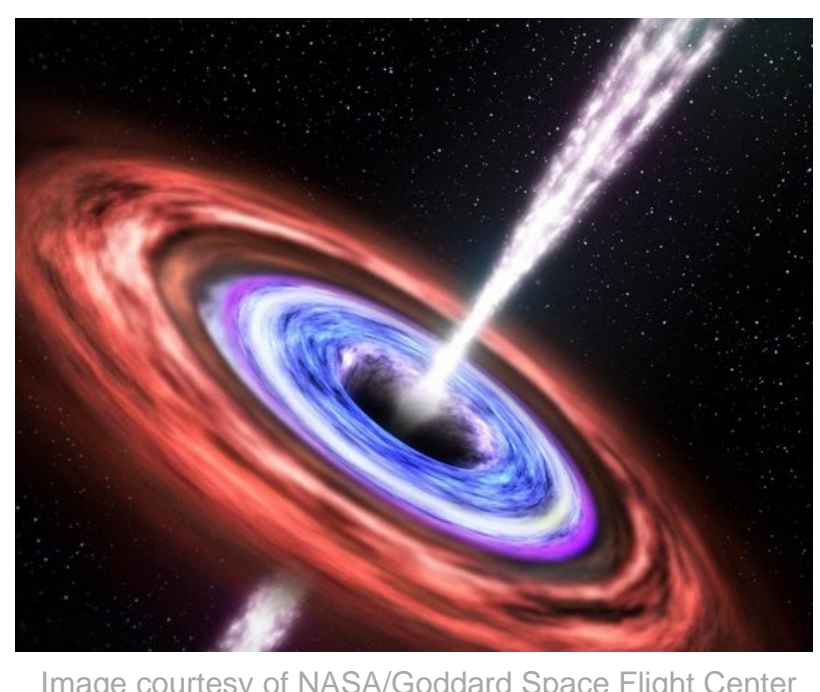

o Most bursts occur at gamma ray and optical energies simultaneously, but there are "gamma-ray orphans," in which a simultaneous optical burst is not present.

o Different models of blazar emissions have different predictions for these orphans. 


\section{Problem Addressed}

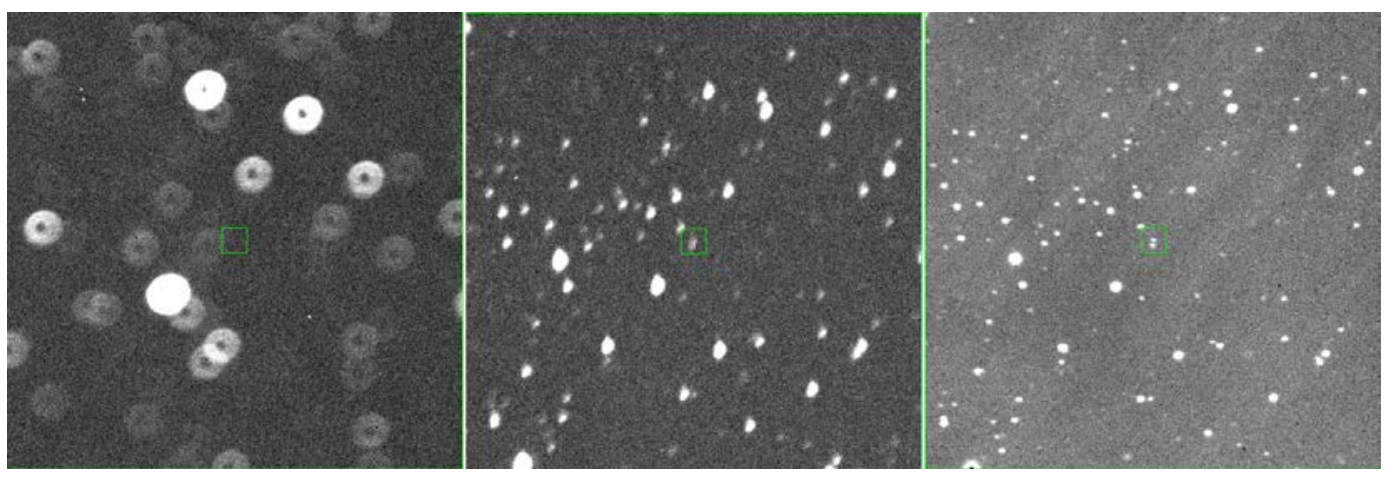

- Investigating the optical variability patterns for blazars tracked by the RAPTOR-S telescope requires the construction of light curves.

- In order to generate these light curves, data must be filtered before processing to ensure quality. 


\section{Methodology}

- Designed 3-step quality filter system for blazar images.

- Coarse filtering using object lists and background brightness

- Calibration of uncalibrated data using astrometry.net

- There layer convolutional neural network trained with 5000 labeled images.

- Implemented automated IRAF photometry pipeline to extract lightcurves from filtered images 


\section{The Neural Network}

- Network uses two convolutional/pooling layers, and a single fully connected layer.

- Input is a 28x28 greyscale image of the object's surroundings.

- Sizes were tested from $8 \times 8$ pixels to the entire $1024 \times 1024$ image, with multiple binning schemes.

- Robustness of network was tested across three different labeling methods and the injection of junk data.

- System achieved screening accuracy of $99 \%$ on validation data. 


\section{Conclusions}

- Example light curve generated by system.

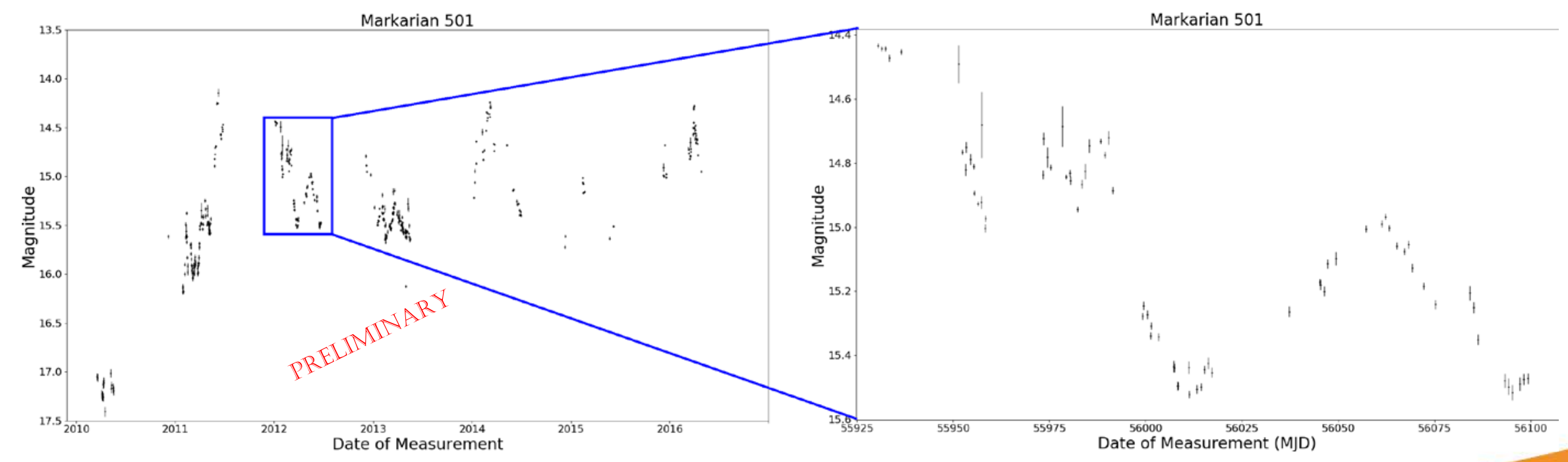




\section{Future Work}

- Future Work:

- Detailed validation of photometric measurements.

- Generate light curves for the entire RAPTOR-S Blazar database.

- Implement system for blazar variability tracking. 